\title{
REVISIONES
}

\section{Modos de interacción como fundamento en la realización de una evaluación formativa no instrumentada}

\author{
Types of interactive dynamics in the implementation \\ of non-written formative assessment \\ Modos de interação como fundamento da realização \\ de uma avaliação formativa não-instrumentada
}

\author{
Morrissette, Joelle ${ }^{a}$ \\ aFaculté des Sciences de L’Éducation, Université de Montréal, Québec, Canadá. \\ Teléfono: 39-40-514 343-6111. \\ Correo electrónico: joelle.morrissette@umontreal.ca
}

\begin{abstract}
RESUMEN
El presente artículo da cuenta de una investigación que documentó las formas en las que se realiza la evaluación formativa no instrumentada en el marco de la interacción entre el profesor y los alumnos durante las actividades en clase. Más precisamente, en esta investigación se examinaron las dinámicas interactivas que tienen lugar durante dichas evaluaciones. Con este fin, se registraron en vídeo clases de 10 profesores de primaria, alternándose con entrevistas individuales, las cuales permitieron a los profesores explicar sus prácticas. Los resultados muestran distintos modos de interacción, tales como ajuste, confrontación, negociación, seducción e humor. Estos dejan ver que la evaluación es un momento privilegiado para la negociación del contrato pedagógico en una clase.
\end{abstract}

Palabras clave: evaluación formativa, modos de interacción, docentes de primaria, socialización, contrato pedagógico.

\begin{abstract}
This research documents the ways in which formative assessment is informally carried out through interactions between teacher and students during classroom activities. Especially, it analyzed the interactive dynamics involved in its implementation. In order to do so, video sequences of teachers were produced in 10 elementary school classrooms with individual interviews aiming to explicit practices. The results demonstrated the presence of different types of interaction, including confrontation, adjustment, dealing, seduction, and humor. Data collected allow us to see that assessment is a privileged time for negotiating the pedagogical contract within the classroom.
\end{abstract}

Key words: formative assessment, types of interaction, primary teachers, socialization, pedagogical contract.

\section{RESUMO}

Apresenta-se uma investigação que documentou as formas nas quais se realiza a avaliação formativa não-instrumentada no marco da interação entre professor e estudantes durante as atividades realizadas em classe. Mais precisamente, examinaram-se as dinâmicas interativas que ocorrem durante tais avaliações. Para esta finalidade, gravaram-se, em vídeo, aulas em 10 professores primários, alternando-se entre entrevistas individuais, as quais permitiram aos professores explicar as suas práticas. Resultados mostram diferentes modos de interação, tais como ajuste, confrontação, negociação, sedução e humor. Tais modos revelam que a avaliação é um momento privilegiado para negociar o acordo pedagógico em uma aula.

Palavras chave: Avaliação formativa, modos de interação, professores primários, socialização, acordo pedagógico. 


\section{INTRODUCCIÓN}

En el momento en que la mayoría de los países occidentales ponen en marcha reformas para mejorar el éxito educativo, la atención se centra en la evaluación formativa de los aprendizajes; el objetivo no es sancionar ni realizar una selección social, sino más bien apoyar la progresión de los aprendizajes. Varios expertos (Black \& Wiliam, 1998; Perrenoud, 1998; Allal \& Mottier Lopez, 2005; Scallon, 2007) sostienen de hecho que la evaluación formativa ofrece posibilidades de mejoría de los procesos de aprendizaje y constituye un elemento clave de la diferenciación pedagógica que permite explorar las dificultades experimentadas por un alumno y elaborar pistas para ayudarlo a regular sus procesos de aprendizaje. Es sobre esta hipótesis que se fundan diversas iniciativas políticas, específicamente en Gran Bretaña, Australia y en Nueva Zelanda, así que programas internacionales, tal como el dirigido por la OCDE (2005). En Quebec, la reforma curricular implementada desde 2001, al apostar sobre un trabajo por competencias, privilegia claramente la función formativa de la evaluación (Gouvernement du Quebec, 2003), específicamente por el carácter de desarrollo de estos objetos de aprendizaje que necesitan un apoyo continuo (Legendre, 2004) y preocupación por las progresiones diferenciadas de los alumnos (Scallon, 2007).

Sin embargo, en Quebec, como en otra parte (por ejemplo, en el cantón de Vaud en Suiza), el debate público alrededor de la evaluación de las competencias se enfocó principalmente en el tema (sensible) de las formas de calificación en los boletines de notas de los alumnos o en el lugar de los conocimientos, tales como lo atestiguan numerosos artículos publicados en los diarios y escritos científicos (Breton, 2011). Uno de los cambios inducidos por la reforma en curso y del que se habla poco en ese debate, es el lugar incrementado del alumno en la evaluación de sus aprendizajes. Tal como lo subraya Le Boterf (2001), la evaluación de las competencias no es una ciencia exacta; es el resultado de un compromiso, de un enfrentamiento de diversos puntos de vista, entre los que el del principal concernido.

Fue con esta visión que encabecé una investigación colaborativa con 10 profesoras de primaria. Apostando sobre los resultados de una investigación anterior en la que documenté sobre todo prácticas de evaluación formativa instrumentadas (Consejo de Investigación en Ciencias Humanas de Canadá, [CRSH], 2005-2008), el enfoque se ha hecho esta vez sobre las que son informales, es decir que se co-construyen dentro de los intercambios entre la profesora y los alumnos en el seno mismo de las actividades diarias (Fondo de investigación de Quebec - Sociedad y Cultura, [FRQSC], 2010-2013). Recordando una perspectiva interaccionista que lleva a proyectar la clase como una micro sociedad en re(composición) continua a través de los intercambios entre actores (Morrissette, Guignon et Demazière, 2011), esta investigación permitió distinguir diferentes modos de interacción que caracterizan dinámicas de regulación apuntadas por la evaluación formativa, entre las que el ajuste, el enfrentamiento, la negociación, la seducción y el humor. En el marco de esta contribución, ejemplifico cómo estos modos se desarrollan en clase a partir de casos específicos que plantean un debate sobre las conductas más o menos (in) determinadas implicadas en la negociación del contrato pedagógico en relación con la socialización escolar. 


\section{DESDE UNA CONCEPCIÓN MECÁNICA A UNA CONCEPCIÓN INTERACTIVA Y SITUADA}

Al cruzar diferentes recopilaciones de escritos sobre la evaluación formativa ( Black y Wiliam, 1998; Allal \& Mottier Lopez, 2005; Morrissette, 2010; Bennett, 2011), se desprende una tendencia en investigación, o sea la de la adopción de una perspectiva más bien instrumental y mecánica (Wininger, 2005; Militello, Schweid \& Sireci, 2010). En varios escritos, la evaluación formativa corresponde a tareas-papel tipo "mini-pruebas" entregadas a finales de etapa y recogidas con un objetivo somático. Esta manera de ajustar la evaluación formativa plantea distintos problemas, entre los que el de reducir los aprendizajes a lo que se mide, evacuando así demasiado rápido la complejidad de los vínculos entre los aprendizajes y su evaluación. Por otra parte, para entender el desarrollo de las competencias en los alumnos, este tipo de uso tradicional de la evaluación conoce límites (Le Boterf, 2001; Perrenoud, 2004), ya que una lógica sumaria no sabría dar cuenta de un conjunto de recursos de diversas naturalezas y de la manera con la que fueron combinados por los alumnos para cumplir con una tarea (Legendre, 2004). De hecho, unos trabajos mostraron que si la opinión de profesoras de primaria acerca de la progresión de los alumnos se apoya sobre este tipo de mini-pruebas, se apoya también sobre el flujo de observaciones e intercambios informales diarios con los alumnos que parecen permitirles entender la complejidad de los aprendizajes (Bell, 2000; Morrissette, 2010).

Otra tendencia dominante del área, y que decae de la primera identificada, está vinculada con una concepción muy mecánica del feedback, o sea de la retroacción proporcionada alrededor de las tareas de evaluación formativa que deberían favorecer la regulación de los aprendizajes. Heredada del behaviorismo, remite a estudios que apuntan a medir los efectos del feedback sobre la progresión de los alumnos que concluyen más a menudo a efectos positivos (Kluger \& DeNisi, 1996; Dawson, Meadows \& Haffie, 2010). Este tipo de investigación que se basa en las primicias de la investigación experimental (pre/ post prueba), reduce la noción de progresión al aumento de un escore en una prueba, lo que, de nuevo, parece constituir una concepción relativamente reductora del aprendizaje.

De hecho, evacua demasiado rápido la cuestión de la interpretación, del ajuste de sentidos, de las dinámicas de regulación interactivas mediatizadas por el lenguaje y los recursos culturales que participan del apoyo a los aprendizajes de los alumnos apuntado por la evaluación formativa.

La importancia creciente de las concepciones más (socio) constructivistas del aprendizaje a lo largo de los 15 últimos años llevó al surgimiento de nuevas perspectivas en investigación (Morrissette, 2009). Investigadores proponen conceptualizaciones que establecen vínculos entre las prácticas de evaluación formativa y la cultura en la que se inscriben, poniendo así de realce las valorizaciones sociales que las afectan (Pryor \& Crossouard, 2008; McMillan, 2010). También fue en esta corriente, y con razón del interés otorgado ahora a los puntos de vista de los especialistas que se desarrolló una concepción amplia e interactiva de la evaluación formativa, como fundamento a trabajos con orientaciones diversas. Por ejemplo, en colaboración con profesores, diferentes estrategias de regulación interactiva fueron documentadas, a menudo bajo un ángulo didáctico (Kasadi, 2005; Mottier Lopez, 2007) o más ampliamente bajo el ángulo del saber-evaluado de los profesores (Bell, 2000; Mertler, 2009; Morrissette, Loye et Legendre, 2011). Algunos trabajos fructíferos del punto de vista de la originalidad de su contribución y del estudio 
de la complejidad del apoyo a los aprendizajes estudiaron las maneras por las cuales el profesor y los alumnos colaboran en clase en un esfuerzo de ajuste de sentido. Por ejemplo, Mottier Lopez (2003) realzó que un sistema entero de convenciones, que se expresa en términos de expectativas y obligaciones mutuales, preside a las estructuras de participación en clase implicadas en las regulaciones interactivas.

Brandta \& Tatsisb (2009) distinguieron diferentes roles adoptados por los principales actores concernidos en el marco de esa colaboración, y la influencia de esos roles en las posibilidades de mejoría de los procesos de aprendizaje. Al adoptar una visión interaccionista y situada de la evaluación formativa, mis trabajos se inscriben en la línea de esas investigaciones. Hasta el momento, la co análisis de las prácticas realizada con profesoras de primaria permitió descubrir su saber-hacer, dando cuenta del trabajo minucioso y etnográfico del profesor quien, tal como el transeúnte de Baudelaire, observa, emite hipótesis provisorias, crea vínculos, cuestiona etc., para darse una representación de la situación de los alumnos frente a los aprendizajes apuntados e intervenir de manera diferenciada (Morrissette et Compaoré, 2013).

En el marco de este artículo, el análisis de la coordinación entre el profesor y los alumnos está reforzado, siendo el enfoque "los juegos de interdependencia" (Postic, 1979) que participan de la puesta en marcha de una evaluación formativa no instrumentada. Dentro de esta visión, y a continuación de los trabajos mencionados anteriormente, el interés se centra en las conversaciones entre el profesor y los alumnos, constituyendo éstas un lugar de ajuste (más o menos armonioso) de los significados en el marco del apoyo a los aprendizajes apuntado por la evaluación formativa. Las explicaciones teóricas que acompañan esta concepción están presentadas en la parte siguiente.

\section{UNA CONCEPCIÓN INTERACCIONISTA Y SITUADA DE LA EVALUACIÓN FORMATIVA}

La evaluación formativa se estudia aquí desde una perspectiva interaccionista y situada que ofrece un marco de análisis de los procesos de interdependencia, de las dinámicas a través de las cuales los actores actúan para coordinarse en una situación (Morrissette, Guignon et Demazière, 2011). Se basa, desde los trabajos de autores asociados con el interaccionismo simbólico -Thomas (1923), Becker (1986, 2006), Strauss (1992)- en una tradición de investigación que incentiva el estudio de los fenómenos sociales bajo el ángulo de las interacciones que vinculan a actores a diario y de las significaciones que cobren en estas interacciones. Se basa también, por analogía prudente, en el trabajo de Laborde (1998) sobre la improvisación que explica más específicamente el contexto de la evaluación formativa no instrumentada, su carácter a la vez indeterminado y previsible.

El concepto de "definición de la situación" (Thomas, 1923) remite a la manera con la que los actores proyectan la situación en la que se encuentran, y entonces a su manera de enmarcarla, a los obstáculos que creen deber enfrentar, a las elecciones que piensan tener, etc. Permite proyectar las prácticas más informales de evaluación formativa entonces consideradas como procesos continuos de definición de la situación que maneja el profesor. Estos procesos interpretativos están hechos de lo que le parece pertinente para entender los problemas a los que se enfrentan los alumnos, por ejemplo, las dificultades experimentadas anteriormente, y que lo lleva a intervenir de cierta manera con ellos para apoyar sus 
aprendizajes. Sin embargo, después de Becker (1986), figura de proa del interaccionismo simbólico, recordaremos que el actuar del actor es función del ajuste de su "definición de la situación" respecto a la de los demás, construyéndose poco a poco el tejido de los intercambios entre compañeros de la interacción a través de las contribuciones mutuas arraigadas en la definición de la situación de cada uno. En ese sentido, es más exacto hablar de una "co definición de la situación" que estaría emergiendo constantemente y negociada en las interacciones en clase, permitiendo a los principales concernidos ajustarse más o menos armoniosamente; según el grado de intercambio de las interpretaciones, puede haber turbulencia en la interacción, conflictos o puntos muertos. Conviene considerar la dimensión temporal de la acción, en función de una de las hipótesis teóricas del interaccionismo simbólico (Strauss, 1992): las maneras por las cuales el profesor y los alumnos se ajustan en un contexto de evaluación formativa están insertadas en conjuntos de condiciones, ellos mismos anteriores, contribuyendo a moldear la situación presente y creando a su vez para las acciones futuras. Por fin, si los compañeros de la interacción pueden compartir un mismo objetivo -el del éxito escolar por ejemplo- sus expectativas e intereses no se convergen necesariamente. En consecuencia, la evaluación formativa no podría reducirse a la regulación de los aprendizajes; también concierne la regulación de las posiciones que nunca están fijadas, osificadas (Strauss, 1992).

Como complemento, también es interesante considerar los trabajos de Laborde (1998) que tratan de los Bertsularis, estos artistas vascos que hacen combates improvisados durante los cuales inventan poemas cantados, mesurados y rítmicos. Para el autor, el arte desplegado por estos improvisadores revela una competencia y un objetivo en situación -lo que puede corresponder a la intención del profesor de apoyar los aprendizajes- que fueron forjados a costa de una familiaridad que se desarrolló a lo largo del tiempo como respuesta a las expectativas de un público. De hecho muestra cómo la conducta del improvisador, su sentido del sincronismo, sus avances, su discernimiento respecto a las elecciones que hace rápidamente etc., se apoyan en las reacciones de la muchedumbre, en un ajuste constante, en suma, en lo que se co construye en situación. El bertsu (el poema cantado), dice el autor, es entonces el producto de una interacción entre un individuo y un público, interacción que entrega de vuelta una capacidad para improvisar. Los trabajos de Laborde (1998) hacen eco a los de Becker (1986) sobre los músicos de jazz que hacen sesiones de jam. Los autores están de acuerdo para decir que la idea de improvisación no remite a la de indeterminación. Para que el combate o la sesión de jam tenga lugar, unas especies de acuerdos previos son necesarios, tales como reglas del juego, no consignadas, sino que presiden a la organización de la improvisación. Entonces habría conductas típicas que regirían las relaciones entre actores, dejándoles cierto margen de actuación. Becker (2006) da el ejemplo de un público conocedor que viene a asistir a un concierto de música clásica: sabe no aplaudir entre dos movimientos, lo que ignoraría un público ignorante de las convenciones de este género artístico. Con otras palabras, convenciones -o "rituales de interacción" (Goffman, 1973) - vienen a enmarcar la improvisación. En esa perspectiva, la interacción entre el profesor y los alumnos que intercambian con el objetivo de co construir una representación de lo que obstaculiza el aprendizaje y de co definir una estrategia que apunta a superarlo, se realiza, según diferentes rituales que designo aquí como "modos de interacción", limitados por expectativas mutuas, reconocidos como habituales por los compañeros. Constituirían una especie de contrato contraído más allá de las palabras en el marco de una cultura de clase. 


\section{VIDEOS Y ENTREVISTAS PARA ANALIZAR LA NEGOCIACIÓN DE LAS POSICIONES}

Capturar esos modos de interacción implicados en la realización de una evaluación formativa no instrumentada representa un desafío en el plan metodológico. Con este objetivo, una investigación colaborativa se hizo con 10 profesoras de primaria (E1, E2, etc.), apostando sobre su discernimiento practico para explicar un objeto de preocupación mutua en contexto. Esta metodología de investigación/formación apunta la aprehensión comprensiva de las maneras de hacer para resaltar el saber-hacer subyacente, a beneficio de la comunidad científica como profesional (Desgagné, 2007). Dos de ellas trabajan en el preescolar, dos en educación física, las seis otras tienen clases del sector regular del primer año al sexto año (de 6 a 12 años). Más precisamente, a partir de vídeos realizados en sus clases respectivas (tres por profesora), identificaron lo que constituye, desde su punto de vista, episodios de evaluación formativa no instrumentada. Durante las entrevistas individuales (tres por profesora), explicitaron la acción y su comprensión de la dinámica con los alumnos a partir de la escucha de esos episodios; con este objetivo, fueron apoyadas por intercambios con la investigadora $(\mathrm{CH})$ que apuntaban a profundizar esa comprensión.

La estrategia analítica se apoyó en la micro sociología de Goffman (1973). A partir de las conversaciones de las entrevistas individuales conducidas, y entonces del análisis que las profesoras hacen de la interacción observada en los extractos que seleccionaron, diversos modos de interacción fueron identificados a través de la definición de la situación por cada profesora, de su comprensión de los significados intercambiados con los alumnos concernidos por los episodios seleccionados. Se prestó una atención especial a la negociación de las posiciones respectivas de los compañeros, o sea a las actividades discursivas llevadas en el marco del tipo de tarea que les ocupaba durante un episodio seleccionado: transformación en relato de una dificultad, argumentación, explicación, cuestionamiento mutuo, etc. En un plan más técnico, la codificación de las informaciones se estribó en un estudio inductivo, necesitando la elaboración del cuadro de análisis unos ajustes sucesivos a medida que hubiera un enfrentamiento con un nuevo discurso, según un principio de saturación (Laperrière, 1997). Estos ajustes permitieron precisar la definición de los modos de interacción identificados, a fin de distinguirlos los unos de los otros, por la integración progresiva de nuevas unidades de sentido. Por ejemplo, cuando la profesora explica un episodio resaltando construcciones consensuales entre ella y un o unos alumno(s), avances de un lado al otro que convergen en una búsqueda de sentido para solucionar un problema de aprendizaje, un código se atribuye para designar un modo llamado de "ajuste"; cuando la profesora comenta un episodio dentro del cual sus intercambios con un o unos alumno(s) están caracterizados por diferencias que se cristalizan alrededor de las tareas que cumplir y de las acciones que realizar para hacerlo, un código se atribuye para designar un modo llamado de "enfrentamiento".

\section{ALGUNOS MODOS DE INTERACCIÓN IMPLICADOS EN LA REALIZACIÓN DE UNA EVALUACIÓN FORMATIVA NO INSTRUMENTADA}

La co construcción de una evaluación formativa siempre estriba en el compromiso improvisado de los compañeros, a lo largo de los intercambios, pero también en rituales 
previsibles y familiares. Es por eso que, los modos de interacción presentados aquí, aunque tienen un carácter muy contextualizado, también son más o menos estables y frecuentes, pudiendo visualizarse en los episodios de clase de todas las profesoras. Así, la investigación lleva a considerar que una evaluación formativa no instrumentada se co realiza sobre la base de diferentes modos de interacción típicos, entre los cuales el ajuste, el enfrentamiento, la negociación, la seducción y el humor. Cada uno de estos modos se define aquí, luego se ejemplifica a partir de un extracto de discurso. La interpretación de cada uno de los casos presentados realza las problemáticas respectivas en la negociación de sentido implicada en la realización de una evaluación formativa.

\subsection{EL AJUSTE}

El "ajuste" constituye un primer modo de interacción identificado en varios episodios de evaluación formativa informal. Observamos una dinámica de ajuste cuando el profesor y el estudiante reúnen sus posiciones respectivas en una búsqueda de sentido compatible con el objetivo de entenderse, darse una representación en común del problema que se plantea y eventualmente cooperar para encontrar pistas de solución. El extracto presentado está sacado de una entrevista con una de las dos educadoras que trabajan en el preescolar.

E1: Me he dado cuenta de que Antoine (5 años) tiene dificultad para expresar lo que piensa; fue lo que entendí de las conversaciones diarias que tuve con él; aquí, en este episodio, debe encontrar un orden para los cuatro dibujos, poco importa cuál orden, lo importante es que los ponga en un orden que produzca una historia coherente; esto es difícil para él, porque lo obliga a traducir con palabras la historia que tiene en mente, ya que debe justificar su elección; aquí observo que está fijando su hoja sin poder decir nada; entonces mi forma de intervenir de manera formativa es ayudándolo a explicitar esa historia probando ideas, de no ser así, no lo consigue; acá le sugiero que se trata de una historia de Halloween y me dice: "no", entonces le pregunto "no ¿por qué Antoine?" ¿Qué te hace decir que no?".

$\mathrm{CH}$ : ¿Tenías otro tipo de historia en mente?

E1: Sí y mire, me repite que ésta no es una; retomo a partir de ahí preguntándole de qué trata la historia; entonces consigue decirme "un baile disfrazado"; cada vez que añade un pequeño trozo de historia, me permite hacerle otra pregunta para ayudarlo a explicar lo que tiene en mente, y a validar la coherencia del encadenamiento de las imágenes; es así que consigo que avance; me ayuda a ayudarlo diciéndome si las ideas que pruebo corresponden a lo que tiene en mente.

Este extracto muestra la cooperación entre la educadora y el alumno que permite que se desprenda un sentido compatible alrededor de la historia imaginada por este último. La educadora utiliza el cuestionamiento para validar pistas y el niño acepta esta manera de hacer, ya que alimenta la dinámica al participar de una retroacción -aunque sucinta- sobre la pertinencia de estas pistas respecto a la historia que tiene en mente. En ese sentido, el intercambio es armonioso, trayendo a cada uno beneficios; también se inscribe en un ritual conocido y aceptado de los dos compañeros. De hecho la dinámica de cuestionamiento forma parte del código de la escuela, y vemos aquí que desde el preescolar, los alumnos ya están acostumbrados a esto. Este episodio muestra que una evaluación formativa se construye de a dos, se co define paso a paso en lo inmediato, pero también que las condiciones de la situación presente están inscritas en una historia, ya 
que la manera de hacer de la educadora estriba en lo que entendió ella de las diferentes conversaciones que mantuvo con Antoine.

Es entonces ese juego de interdependencia arraigado que le permite asegurarse que el razonamiento lógico del niño es válido, o sea un objeto de evaluación formativa propia de algunas competencias apuntadas en el preescolar, entre los que "construir su comprensión del mundo" y "comunicar utilizando los recursos del idioma" (Gobierno de Quebec, 2001).

\subsection{EL ENFRENTAMIENTO}

Un segundo modo de interacción implicado en los episodios de evaluación formativa no instrumentada es el "enfrentamiento", o sea un ritual durante el cual el profesor y el alumno reúnen sus posiciones respectivas en un movimiento de oposición; resulta un conflicto que supera el ajuste de sentido, tal como lo vimos en el ejemplo anterior. Este extracto de discurso expone la interpretación que hace una profesora de tercer año de sus intercambios con una de sus alumnas, haciendo ver este modo de interacción.

E7: Clara es una niña muy emotiva: si no consigue hacer su tarea, llora, luego lo va a dejar todo; en el inicio del año, la dejaba arreglárselas sola, para trabajar su autonomía frente a sus aprendizajes, pero me di cuenta de que no estaban los resultados deseados; refunfuñaba, lloraba, iba a llamar la atención de los demás, etc.; en el vídeo, durante este episodio, vemos que empieza a frustrarse respecto a su trabajo; no lo consigue; como no sé si es porque no ha entendido las instrucciones, le vuelvo a explicar, le hago una demostración e insisto para que retome su tarea enseguida hasta que lo logre.

CH: De acuerdo.

E7: Porque me digo que si lo consigue, se va a desbloquear y desarrollar una relación más positiva con el trabajo; entonces, persevero con mi petición; pero se niega a retomar el trabajo, las mejillas se vuelven rojas, no me contesta o solo me contesta cosas no pertinentes, únicamente para provocarme; mi evaluación me lleva a identificar un problema de actitud; intervengo insistiendo para que retome la tarea a fin de que viva un éxito, pero cuanto más insisto, cuanto menos colabora; voy a tener que volver a estudiar mi forma de hacer con ella.

Al contrario del modo de ajuste visto previamente, donde la educadora y el niño cooperaban para darse una representación compartida susceptible de superar el obstáculo de aprendizaje, de una manera relativamente rápida y armoniosa, constatamos aquí una turbulencia en la interacción, llevando incluso el intercambio a un punto muerto. La evaluación que hace la profesora de la situación lleva a dos intervenciones formativas no fructuosas, quedándose con su posición respectiva los compañeros: ni el uno ni el otro cede, siendo irreconciliables por el momento sus reivindicaciones respectivas. La profesora explica su postura diciendo que su evaluación informal diaria del desarrollo de Clara la ha llevado a identificar que es un problema de actitud que obstaculiza sus aprendizajes. Sin embargo, por el momento, sus tentativas para solucionarlo, que van en el sentido de una especie de insistencia y enfoque sobre la tarea que cumplir con el objetivo de llevarla a perseverar a pesar de las dificultades, no encuentran una apertura favorable. Tal como otros modos de interacción también lo dejan ver, constatamos entonces que hay un frágil equilibrio entre el compromiso personal de la profesora para enseñar y la calidad de su relación con el niño. 


\subsection{LA NEGOCIACIÓN}

El cierto "inmovilismo mutuo" destacado del extracto anterior se opone a un modo de interacción que implica concesiones de ambas partes, juzgadas satisfactorias para las dos partes implicadas. Así un tercer modo identificado concierne a una profesora y un alumno que reúnen sus posiciones respectivas en un movimiento de compromiso, de conciliación; es designado como una "negociación", acercándose al significado común dado a la palabra "negociación". Para ejemplificar su desarrollo en la interacción, presento la narración de un episodio implicando a una educadora del preescolar y dos alumnos que están asignados a un taller que no parece entusiasmarlos.

E6: Mathis y Kevin no aprecian para nada talleres donde deben sentarse y escribir concentrados; los observo desde el inicio del año: no es muy fácil para ellos; en este episodio, debían hacer una banda decorativa sobre el tema de Halloween; era la continuación de una tarea de prelectura que no les gustó, que no permitía moverse, estar en acción.

CH: Y la pequeña banda, ¿era una actividad que hacer cortando?

E6: No; había que dibujar una serie de pequeños fantasmas con lápices de color, una banda según cierta secuencia en un trozo de papel; el problema era que jugaban con los lápices en vez de hacer la banda; en ese momento, mi evaluación me lleva a pensar que quizá no entendieron la actividad; averigüé: no era el caso; entonces pregunté "¿es porque no tienen ganas de hacer ese taller?"; me contestaron que sí; entonces decidí ceder en parte al permitirles que eligieran el tamaño de la banda; hicieron una muy corta y les dije que era suficiente; de hecho prefería un trabajo minimalista que me permite ver si entienden la idea de secuencia que nada; por lo menos, ¡lo hicieron!

El extracto muestra claramente las negociaciones entre los compañeros del intercambio que tienen a primera vista intereses divergentes, pero que obran para encontrar una solución mutuamente aceptable. De hecho, esos jóvenes alumnos parecen tener más ganas de jugar, de moverse, más que de hacer la demostración de su comprensión de una secuencia, un aprendizaje básico en matemáticas. En cuanto a la profesora, ella ejerce un discernimiento en situación y está atenta a vigilar si ese aprendizaje está asimilado.

Hecha la negociación, o sea aceptar que los alumnos produzcan una banda corta, satisface a los compañeros que aceptan perder un poco respecto a sus primeros intereses, como condición necesaria a la evaluación formativa de los aprendizajes. Desde cierto punto de vista, podríamos considerar de que se trata de una especie de trueque, lo que Woods (1990) llamaría un mecanismo de supervivencia de la profesora. Sin embargo, como esta última no hace concesiones sobre los aprendizajes y su evaluación, una interpretación más fecunda iría más bien en el sentido de ver su manera de gestionar la relación pedagógica.

\subsection{LA SEDUCCIÓN}

La "seducción" es el cuarto modo de interacción identificado en ciertos episodios de evaluación formativa no instrumentada. Implica juegos socio afectivos a los cuales se entregan la profesora y un alumno, derivándoles por un momento, del objetivo de apoyo a los aprendizajes de la evaluación formativa. El extracto de una entrevista permite distinguir 
esta dinámica a través de la narración que una profesora de educación física hace de un episodio implicando a un grupo de alumnos de sexto año, entre los que uno en especial (Jonathan). Es emblemático de varios episodios seleccionados por las profesoras que trabajan en distintos niveles de enseñanza.

CH: Si entendí bien, los alumnos deben circular a través de seis estaciones y realizar desafíos (motores) en cada una de ellas: abalanzarse sobre el pequeño trampolín ejecutando una figura, saltar sin que la soga esté tocada, caminar sobre el cilindro, etc.

E2: Es exactamente esto.

$\mathrm{CH}$ : En esta escena precisa, veo que dice algo a un alumno.

E2: Sí, porque en educación física, la aprobación, decir "está bonito", es muy importante; los alumnos ven que tienen cada uno mi atención en un $100 \%$ por un momento.

CH: De acuerdo.

E2: Eso les motiva, les anima a seguir.

CH: Aquí, ¿interviene más específicamente con...?

E2: Jonathan; al circular, vi que no conseguía caminar en el cilindro porque se inclinaba demasiado hacia adelante; entonces intervengo prestándole atención, valorizando sus esfuerzos, destaco sus mejorías delante de los demás; lo vemos bien en el vídeo; busca constantemente mi mirada y mi aprobación; se ejercita de manera seria únicamente cuando me quedo en esta estación para mirarlo ejercitarse; aquí está orgulloso porque lo felicito; consigo obtener lo que quiero con él de esta manera, prestándole atención y haciéndole cumplidos.

La narración de esta escena muestra una seducción mutua que se traduce de manera diferente en los compañeros. Para la profesora, se ejercita valorizando al alumno, prestándole atención, y reconociéndolo; para el alumno, es más bien actuando conforme a las expectativas: "se ejercita de manera seria". Sin embargo, cuando las posiciones de cada uno se reúnen de esa manera, en el momento, las interacciones no convergen hacia la idea de codefinir una representación similar del problema experimentado por el alumno ni destacan pistas susceptibles de ayudarlo a superarlo; no participan directamente de la regulación de los aprendizajes.

Las interacciones se dirigen más bien hacia juegos socio afectivos que trabajan la relación pedagógica indirectamente. Además, este extracto sugiere cierto modo de hacer implícito en la dinámica en clase, que Kounin (1977) llamaría un efecto de "reverberación". Al realzar delante de sus compañeros de clase, las mejorías de Jonathan mientras le esté prestando atención, se asegura de que entiendan que una conducta de conformidad está recompensada.

Así, en este extracto, este modo de interacción implica más que a los dos compañeros: los demás alumnos forman parte de esa regla más o menos tácita de la escuela.

\subsection{EL HUMOR}

El humor es un quinto modo de interacción implicado en la realización de una evaluación formativa informal. Se observa en extractos en los que las profesoras hacen valer que ellas que sus alumnos jueguen con sus posiciones respectivas para bromear, molestar, incluso crear una situación absurda que da risa. Por ejemplo, es ese modo de interacción que parece vincular a una profesora de primer año con una alumna que experimenta problemas para empezar una tarea de comprensión lectora. 
CH: ¿No había entendido bien las instrucciones?

E5: Me he dado cuenta, observando con el tiempo a Jennifer, cuestionándola, que a menudo sabe qué hacer, pero no siempre le interesa; más bien quiere hacer lo que le da la gana y no lo que he previsto para los talleres de aprendizaje; pero cuando lo presento de otra forma, contesta a lo que le pido; es una forma de intervenir de manera formativa, específica de ella; no debo enfrentarla y exigir que haga esta tarea; esto no funciona con ella.

CH: ¿Entonces, cuál es tu manera de intervenir con ella, de manera específica? Veo en la pantalla que la miras mucho.

E5: Sí, le quito su lápiz porque veo que no piensa en lo que debe hacer; está jugando; no le interesa; de hecho, actué de manera humorística.

$\mathrm{CH}$ ¿De qué manera?

E5: Dije "la niña en la historia, ¿qué le gustaba? ¿el refri? ¿la cama? ¿el árbol de navidad?"; de hecho estas pistas son propuestas absurdas, sin vínculo con la historia, pero ves cómo Jennifer se ríe; entonces me contesta "ipues no, no era lo que le gustaba!".

$\mathrm{CH}:$ ¿Eliges el humor más que el enfrentamiento?

E5: Sí, evito el conflicto, me he dado cuenta de que cuando le doy risa, se compromete más con la tarea.

CH: Vemos en el vídeo que poco después, deja de trabajar.

E2: Pasa a menudo; un poco más lejos, al dar cuenta de que lo dejó, vuelvo hacia ella, y es ella que me hace una broma alrededor de la historia, la cual alimento, y ise pone de nuevo a trabajar!

El humor parece constituir para esta profesora una estrategia que utiliza para hacer más atractivo el taller de aprendizaje. Pero el alumno no solo es un receptor pasivo de este humor: el final del extracto lleva a entender que invita ella misma a la profesora a este modo.

Tratando de los no-dichos o de la cara escondida del trabajo de profesor, Perrenoud (1996) evidencia que para instruir, se necesita, de alguna manera, captar la atencion y la buena voluntad, encontrar el método de que "pase la píldora" diríamos simplemente, frente a saberes y saber-hacer escolares por los cuales los alumnos pueden no encontrar sentido.

En esta perspectiva, dice el autor, el humor es una manera de seducir que es un poderoso motor para el aprendizaje. Varios alumnos asocian el saber con la persona que lo representa, y cuando ésta tiene humor, los aprendizajes más áridos se adquieren por transferencia. Aquí, la profesora consigue que se interese Jennifer por la tarea de comprensión lectora -el contenido de aprendizaje- favoreciendo una transferencia por el juego humorístico.

\section{LA EVALUACIÓN FORMATIVA INFORMAL COMO LUGAR DE NEGOCIACIÓN DEL CONTRATO PEDAGÓGICO}

La evaluación formativa no instrumentada es una actividad en parte improvisada que se juega en el cruce de las relaciones de interdependencia implicando una dinámica de compromiso y ajuste, según modos de interacción estribando en normas, referencias e intereses más o menos convergentes; los resultados presentados son un buen ejemplo. Éstos realzan que ese tipo de evaluación es un lugar privilegiado para la negociación del contrato pedagógico y que la relación entre el profesor y el alumno es el elemento clave. 
Una dinámica de evaluación formativa no instrumentada empieza por el hecho de que una profesora observa que un problema se plantea a uno o unos alumnos, y es a través de un intercambio que permite distinguirlo y pensar en las soluciones que se negocia el contrato pedagógico. El estudio de la interacción en los casos presentados subraya que esta negociación implica más que una relación con el saber y el error. Incluye también problemáticas de competencias, de beneficios y pérdidas, de recompensas, de reconocimientos, de reivindicaciones etc., tantos elementos que forman parte de la socialización escolar, reconoce Perrenoud (1994). Por ejemplo, el caso del ajuste puede acercarse favorablemente a problemáticas expresándose en términos de ganancias mutuas, el de la seducción a problemáticas de reconocimiento mutuo. Además, la investigación lleva también a evidenciar que la negociación del contrato pedagógico pone en marcha procesos que participan también del desarrollo de la acción, aquí vinculada con el apoyo a los aprendizajes apuntado por la evaluación formativa: la identificación de los recursos y obstáculos a tomar en cuenta, la negociación de una posición viable que juega sobre su compromiso y el del otro, el trabajo de la "cara" en términos de elaboración, de proyección y de protección de la imagen de sí y de la del otro (Goffman, 1973), etc.

Así, por una parte, todas estas variables, hacen cada evaluación formativa no instrumentada única. Su práctica se improvisa según los "ingredientes" del contexto y se basa en un compromiso y un posicionamiento negociada in situ, a partir de un arreglo interactivo incesante y flexible. Como lo muestran los casos estudiados desde el punto de vista de las profesoras, los principales concernidos cooperan adentro de un marco donde pueden actuar. Cuando uno de los compañeros adopta cierto posicionamiento, el otro se coloca como complemento más o menos fecundo, busca negociarse una posición viable tomando en cuenta su propio proyecto -que puede diferir del previsto (por la institución escolar)- sus intereses, contribuyendo así él también a la definición de la situación.

El caso de la negociación, exponiendo como la profesora y los alumnos ceden un poco desde la postura inicial, muestra esa flexibilidad que confiere parte de indeterminación en cuanto a la realización de una evaluación formativa no instrumentada. Cada caso es especial y la acción que llevar está co gestionada, ajustada según los recursos y obstáculos del contexto, así que las problemáticas respectivas de cada uno de los compañeros. Por otra parte la evaluación formativa no instrumentada también es en parte previsible, ya que vinculada con condiciones situadas relativamente convencionales, constituidas de sistemas de expectativas mutuas familiares en relación con las maneras de encargarse de los problemas sufridos por los alumnos, tales como formatos esperados de participación en la regulación de los aprendizajes apuntada por la evaluación formativa (Mottier Lopez, 2003).

De hecho, como lo sugieren los trabajos de Laborde (1998) o de Becker (1986, 2006), los intercambios entre los compañeros son eventos muy organizados en razón de su competencia social que les confiere un conocimiento de las reglas de la interacción y que les permite estimar con bastante precisión lo que sus propias conductas están susceptibles de engendrar en los demás. Por ejemplo, el caso de la seducción ejemplifica una regla más o menos explícita del contrato pedagógico (Jeffrey, 2010), cuyas consecuencias relativamente previsibles en términos de conductas mutuas son bien conocidas por los compañeros de la interacción: valorizar la "buena" conducta de un alumno enseña a sus pares que la conformidad está animada y recompensada, sobre todo por atención y señales de reconocimiento de parte del profesor. 


\section{CONCLUSIÓN}

Las competencias de los alumnos se desarrollan principalmente en relaciones intersubjetivas que evolucionan con el tiempo, en vínculo con interacciones situadas en los contextos precisos de su desarrollo. Los resultados de esta investigación subrayan el hecho de que estos contextos se tejen de diversos modos de interacción o "microprocesos sociales" (Strauss, 1992) que participan al apoyo del desarrollo de sus competencias, apuntado por la práctica de una evaluación formativa continua.

Los casos presentados en el marco de esta contribución para ejemplificar esos modos de interacción muestran sobre todo que la cuestión de la relación pedagógica es central para las profesoras, al lado de los intercambios simbólicos que establecen una relación con el saber.

Respecto a eso, los modos de interacción que participan del apoyo a los aprendizajes apuntado por la evaluación formativa pueden constituir un marco de interpretación para la práctica, según el método de los casos (Mucchielli, 1992; Passeron et Revel, 2005).

Durante la formación inicial, casos vinculados con los episodios seleccionados por las profesoras pueden ser presentados a practicantes novicios para valorizar métodos más informales de evaluación formativa llamándoles la atención en situaciones interactivas con un potencial de aprendizajes.

Durante la formación continua, estos mismos casos pueden permitir a grupos de profesores "escenificar" su práctica interactiva de apoyo a los aprendizajes y cuestionarla a la luz de las problemáticas de los contextos particulares en los cuales trabajan.

Por otra parte, la identificación de los modos de interacción en el seno de la evaluación formativa no instrumentada constituye, para la investigación, una puerta de entrada para estudiar la complejidad de su práctica.

Se podría seguir examinando la coordinación de las actividades de los principales compañeros, la negociación del contrato pedagógico a la cual se dedican, especialmente bajo dos ángulos.

1) Convendría estudiar con más atención las relaciones de poder que trabajan esta negociación-persuasión, llamada a la autoridad, coerción etc., ya que la forma escolar (Vincent, 1980) está marcada por una relación con la norma, y la evaluación, que sea formativa o no, es el mecanismo principal de apropiación y de mantiene de esta norma. Si, como lo dice Strauss (1992), la negociación es uno de los métodos para "obtener que las cosas se hagan" (p. 252), la relación de poder interviene en la representación que está co construida por el problema sufrido por un alumno, en las pistas desarrolladas para solucionarlo y en sus repercusiones para los principales concernidos.

2) La negociación del contrato pedagógico también se debe examinar bajo un ángulo más macroscópico. De hecho, como lo hace valer Perrenoud (1998), la evaluación formativa de naturaleza cualitativa pone en juego la progresión de los alumnos en el sistema escolar, y entonces su integración en la cultura que está valorizada. En esta visión, este microanálisis debería ser sostenido por un ángulo de análisis que no se limite al perímetro de la clase, que abraza de manera más amplia. De hecho el análisis realizado en el marco de esta contribución se vinculó con el contexto de negociación cara a cara (Goffman, 1973), con sus problemáticas específicas, dejando 
en la sombra el marco estructural que influye en los casos presentados y que moldea también la realización de una evaluación formativa no instrumentada.

Un ángulo ampliado permitiría entender mejor el conjunto de las interacciones que participan de este juego de arreglos, de compromisos, de acuerdos o de diferencias entre los compañeros, en la perspectiva ya anticipada por Pryor \& Crossouard (2008) que, apoyándose sobre un modelo de evaluación formativa informal arraigada en una teoría de la actividad, conciben la clase como una comunidad de práctica en la cual se negocian más o menos armoniosamente los roles, las identidades, las reglas, el sentido de las tareas y criterios de evaluación etc.

Los autores hacen valer lo que se valoriza socialmente entre en la clase, especialmente por el currículo y los libros escolares, y que las relaciones de poder se reproducen en los que se considera como aprendizajes legítimos y en las solicitudes de evaluación certificativa a través de los que se opera una especie de mediación del apoyo a los aprendizajes.

Los modos de aprendizajes expuestos en el marco de esta contribución son apoyos empíricos a su teorización cuyo examen debe proseguirse a la luz de dimensiones políticas y estructurales que afectan la escuela, o sea la evaluación de los aprendizajes que moldean la acción de apoyo de los profesores.

\section{AGRADECIMIENTOS}

La autora desea expresar su gratitud hacia Sylvie Guignon y Marjorie Vidal por los comentarios emitidos sobre las versiones anteriores del artículo que han permitido mejorarlo.

\section{REFERENCIAS}

Allal, L. \& Mottier Lopez, L. (2005). Formative assessment: Improving learning in secondary classrooms. Paris: OCDE.

Becker, H. S. (2006). Les mondes de l'art. Paris: Flammarion. (1986). Doing things together. Evanston, IL: Northwestern University Press.

Bell, B. (2000). Formative assessment and science education: A model and theorizing. In R. Millar, J. Leach \& J. Osborne (Eds.), Improving science education: The contribution of research (pp. 48-61). Philadelphia, PA: Open University Press.

Bennett, R. E. (2011). Formative assessment: A critical review. Assessment in Education: Principles, Policy and Practice, vol. 18 (1), 5-25.

Breton, P. (2011). Les enseignants s'inquiètent. La Presse, A8. Récupérée à partir de http:// www.lapresse.ca/actualites/education/201206/16/01-4535567-bulletin-unique-les-enseignants-sinquietent.php

Black, P. \& Wiliam, D. (1998). Assessment and classroom learning. Assessment in Education: Principles, Policy and Practice, vol. 5 (1), 7-74.

Brandta, B. \& Tatsisb, K. (2009). Using Goffman's concepts to explore collaborative interaction processes in elementary school mathematics. Research in Mathematics Education, vol. 11 (1), 39-55.

Dawson, D. L., Meadows, K. N. \& Haffie, T. (2010). The effect of performance feedback on student help-seeking and learning strategy use: Do clickers make a difference? The Canadian Journal for the Scholarship of Teaching and Learning, vol. 1 (1), 1-20. 
Desgagné, S. (2007). Le défi de coproduction de savoir en recherche collaborative: Autour d'une démarche de reconstruction et d'analyse de récits de pratique enseignante. Dans M. Anadon (Dir.), La recherche participative: Multiples regards (pp. 89-116). Québec: Presses de l'Université du Québec.

Goffman, E. (1973). La mise en scène de la vie quotidienne. Paris: Minuit.

Gouvernement du Quebec. (2003). Politique d'évaluation des apprentissages: Formation générale des jeunes, formation générale des adultes, formation professionnelle (No 03-00062). Québec: Ministère de L'Éducation.

(2001). Programme de formation de l'école québécoise. Éducation préscolaire, enseignement primaire ( $N^{o}$ 01-00374). Québec: Ministère de L'Éducation.

Jeffrey, D. (Coord.). (2010). Cultures \& Sociétés, $N^{\circ}$ 16: Enseigner, c'est séduire. Paris: L'Harmattan.

Kasadi, C. (2005). Exploration des pratiques de professeurs des mathématiques du secondaire à l'égard de l'évaluation formative en mathématiques. Thèse de Doctorat. Québec: Université du Québec à Trois-Rivières - l'Université du Québec a Montréal.

Kluger, A. N. \& DeNisi, A. (1996). The effects of feedback interventions on performance: A historical review, a meta-analysis, and a preliminary feedback intervention theory. Psychological Bulletin, vol. 119 (2), 254-284.

Kounin, J. S. (1977). Discipline and group management in classrooms. New York: Holt, Rinehart and Winston.

Laborde, D. (1998). Enquête sur l'improvisation. Dans M. De Fornel et L. Quere (Dirs.), La logique des situations. Nouveaux regards sur l'écologie des activités sociales (pp. 261-299). Paris: EHESS.

Laperrière, A. (1997). Les critères de scientificité des méthodes qualitatives. Dans J. Poupart, J.-P. Deslauriers, L.-H. Groulx, A. Laperriere, R. Mayer et A. Pires (Dirs.), La recherche qualitative. Enjeux épistémologiques et méthodologiques (pp. 365-388). Montréal: Gaétan Morin.

Le Boterf, G. (2001). Construire les compétences individuelles et collectives. Paris: Eyrolles.

Legendre, M.-F. (2004). Approches constructivistes et nouvelles orientations curriculaires. D'un curriculum fonde sur l'approche par objectifs à un curriculum axe sur le développement de compétences. Dans P. Jonnaert et D. Masciotra (Dirs.), Constructivisme et choix contemporains. Hommage à Ernst von Glasersfeld (pp. 53-91). Montreal: PUQ.

McMillan, J. H. (2010). The practical implications of educational aims and contexts for formative assessment. In H. L. Andrade \& G. J. Cizek (Eds.), Handbook of formative assessment (pp. 41-58). New York - London: Routledge.

Mertler, C. A. (2009). Teachers' assessment knowledge and their perceptions of the impact of classroom assessment professional development. Improving School, vol. 12 (2), 101-113.

Militello, M., Schweid, J. \& Sireci, S. G. (2010). Formative assessment systems: Evaluating the fit between school districts' needs and assessment systems' characteristics. Educational Assessment, Evaluation and Accountability, vol. 22 (1), 29-52.

Morrissette, J. et Compaore, G. (2013). Le savoir-faire enseignant sur l'évaluation formative informelle. Formation et Profession: Revue Scientifique Internationale en Éducation, vol. 20 (3), 54-64.

Guignon, S. et Demaziere, D. (2011). De l'usage des perspectives interactionnistes en recherche. Recherches Qualitatives, vol. 30 (1), 1-9.

Loye, N. et Legendre, M.-F. (Dirs.). (2011). Autour du savoir-évaluer des enseignantes et des enseignants en salle de classe. Mesure et Évaluation en Éducation, vol. 34 (3), 1-4; 161-164. (2010). Manières de faire l'évaluation formative des apprentissages: Analyse interactionniste du savoir-faire d'enseignantes du primaire. Sarrebruck, CH: Les Éditions Universitaires Européennes.

(2009). La portée d'une perspective socioculturelle de l'évaluation formative: Vers l'élargissement d'une conceptualisation. Mesure et Évaluation en Éducation, vol. 32 (2), 1-23. 
Mottier Lopez, L. (2007). Régulations interactives situées dans des dynamiques de microculture de classe. Mesure et Évaluation en Éducation, vol. 30 (2), 23-47.

. (2003). Les structures de participation de la micro-culture de classe dans une leçon de mathématiques. Revue Suisse des Sciences de L'Éducation, vol. 25 (1), 161-183.

Mucchielli, A. (1992). La méthode des cas: Connaissance du problème. Paris: ESF.

OCDE (2005). L'évaluation formative: Pour un meilleur apprentissage dans les classes secondaires. Paris: Centre pour la recherche et l'innovation dans l'enseignement - Organisation de coopération et de développement économiques.

Passeron, J.-C. et Revel, J. (2005). Penser par cas. Paris: EHESS.

Perrenoud, Ph. (2004). Évaluer des compétences. L'Éducateur, (Numéro Spécial). Récupérée à partir de http://www.unige.ch/fapse/SSE/teachers/perrenoud/php_main/php_2004/2004_01.html (1998). L'évaluation des élèves. De la fabrication de l'excellence à la régulation des apprentissages: Entre deux logiques. Bruxelles: De Boeck. (1996). Enseigner: Agir dans l'urgence, décider dans l'incertitude. Savoirs et compétences dans un métier complexe. Paris: ESF. (1994). La formation des enseignants entre théorie et pratique. Paris: L'Harmattan.

Postic, M. (1979). La relation éducative. Paris: PUF.

Pryor, J. \& Crossouard, B. (2008). A socio-cultural theorization of formative assessment. Oxford Review of Education, vol. 34 (1), 1-20.

Scallon, G. (2007). L'évaluation des apprentissages dans une approche par compétences. Bruxelles: De Boeck.

Strauss, A. L. (Dir.) (1992). La trame de la négociation: Sociologie qualitative et interactionnisme. Paris: L'Harmattan.

Thomas, W. I. (1923). The unadjusted girl: With cases and standpoint for behavior analysis. Boston: Brown and Co.

Vincent, G. (1980). L'école primaire française. Étude sociologique. Lyon: Presses Universitaires de Lyon - Editions de la Maison des Sciences de l'Homme.

Winiger, S. R. (2005). Using your tests to teach: Formative summative assessment. Teaching of Psychology, vol. 32 (3), 164-166.

Woods, P. (1990). Teacher skills and strategies. London - New York: Falmer Press. 